\title{
Natural history of prostatic lesions on serial multiparametric magnetic resonance imaging
}

Samrad Ghavimi ${ }^{1}$; Hamidreza Abdi ${ }^{1}$; Jennifer Waterhouse ${ }^{2}$; Richard Savdie ${ }^{1}$; Silvia Chang $^{2}$; Alison Harris ${ }^{2}$; Lindsay Machan ${ }^{2}$; Martin Gleave ${ }^{1}$; Alan So ${ }^{1}$; Larry

Goldenberg ${ }^{1}$; Peter C. Black ${ }^{1}$

${ }^{1}$ Department of Urologic Sciences; ${ }^{2}$ Department of Radiology; University of British Columbia, Vancouver, BC, Canada

Cite as: Can Urol Assoc J 2018 April 6; Epub ahead of print.

http://dx.doi.org/10.5489/cuaj.4859

Published online April 6, 2018

\section{$* * *$}

\section{Abstract}

Introduction: The natural history of prostatic lesions identified on multiparametric magnetic resonance imaging (mpMRI) is largely unknown. We aimed to describe changes observed over time on serial MRI.

Methods: All patients with $\geq 2$ MRI studies between 2008 and 2015 at our institution were identified. MRI progression was defined as an increase in Prostate Imaging Reporting and Data System (PI-RADS; version 2) or size of existing lesions, or the appearance of a new lesion PIRADS $\geq 4$. Patients on active surveillance (AS) were analyzed for correlation of MRI progression to biopsy reclassification.

Results: A total of 83 patients (54 on AS and 29 for diagnostic purposes) underwent serial MRI, with a mean interval of 1.9 years between scans. At baseline, 115 lesions (66 index, 49 non-index) were identified. Index lesions were more likely than non-index lesions to increase in size $\geq 2 \mathrm{~mm}$ (36.2 vs. $7.3 \%$; $=0.002$ ). Overall progression was more likely to be seen among the index cohort (34.8 vs. $7.6 \%$; $<<0.001$ ). New lesions with PIRADS $\geq 4$ were seen on second imaging in 13 (16.5\%) men, and became the index lesion in 29 cases (34.9\%). Eighteen men on AS showed evidence of MRI progression (five with new lesions, 13 with progression of a previous lesion). Biopsy reclassification was present in three men (16.7\%) with and seven men without MRI progression (19.4\%). Conclusions: Overall changes in size and PIRADS scores of index lesions on MRI were small. New lesions were common, but usually did not alter management. 


\section{Introduction}

For a long time prostate cancer (PCa) has been the only cancer in the body diagnosed by blind biopsy without visualization of a suspicious lesion $(1,2,3)$. This paradigm, however, is rapidly changing with the advent of multiparametric magnetic resonance imaging (MRI). Technologic advances, including stronger magnets, diffusion-weighted imaging and dynamic contrast enhancement, have greatly improved the clinical utility of MRI for the diagnosis of prostate cancer $(2,4)$. It is now used frequently in the context of active surveillance, as well as in patients with persistent suspicion of prostate cancer despite a prior negative biopsy $(1,5)$. MRI is even being studied prior to biopsy, which is perhaps the last frontier in prostate imaging (6). The attractiveness of MRI is the potential to reduce the need for prostate needle biopsy, which is invasive and is associated with significant complications (7-10). However, the imperfect negative predictive value of MRI for significant prostate cancer prevents us from leaving out biopsy in men with a normal MRI $(2,3)$.

The increased use of multiparametric MRI for prostate cancer detection raises some novel questions that we have not had to address previously. One key unanswered question is the utility of sequential MR imaging of the prostate. Little is known about the natural history of prostate lesions, and the likelihood of identifying new lesions over time. There is also a lack of literature available to guide the frequency at which serial MRI should be performed on patients with suspicious lesions deemed worthy of followup imaging.

We have adopted widespread use of multiparametric MRI of the prostate since 2010. Most patients on active surveillance undergo MRI, and many after prior negative biopsy if there is a persistent concern for possible prostate cancer. Here we have used our experience with MRI to study the natural history of prostate lesions over time on serial imaging.

\section{Methods}

Study population

In this retrospective study, we reviewed all patients diagnosed with PCa between January 2008 and January 2015. Of the 754 men who underwent mpMRI, 83 were identified who underwent multiple scans of their prostate, including 2 scans in 75,3 scans in 7 and 4 scans in 1 patient. Patients with multiple scans had their most recent scan compared to their original one, to assess for progression. From this cohort, 54 men were identified who were on AS, while 29 were being scanned for diagnostic purposes without a confirmed cancer (“diagnosis cohort”). In these latter patients, the indication for MRI was a persistently elevated (or a rising) PSA and/or suspicious findings on a DRE. The 
selection of MRI over repeat biopsy was made at the discretion of the practicing urologist and was not based on reproducible criteria.

\section{Multiparametric MRI}

MRI was acquired with the use of a 1.5T scanner (Siemens) with an 18-phased-array body surface coil. No endorectal coil was used for this study. Sequences from the MRI were produced via high-resolution T2-weighted imaging, diffusion weighted imaging (DWI), along with high-temporal dynamic contrast enhancement (DCE) imaging. DWI was done with $b$ values of 50,500, and 1,000, with an apparent diffusion coefficient (ADC) map produced by b500 and b1000 images. DCEs were acquired through the intravenous administration of $0.1 \mathrm{mmol} / \mathrm{kg}$ of intravenous gadobutrol (Gadovist; Bayer Schering Pharma) by a power injector with 60 data acquisitions at a temporal resolution of 3.97 seconds.

All scans were re-reviewed and evaluated by a single radiologist with prostate MRI experience, for 2 key purposes: (1) to ensure consistent results given that operatordependent heterogeneity may exist, and (2) to take into consideration the PI-RADs 2.0 scoring system that was established in November 2014 (11). Lesions from the first scan were identified as either index, or non-index lesions. The index lesion was considered the dominant lesion with the greatest potential to harbour significant prostate cancer $(12,13)$. We defined the index lesion as the lesion with the highest PI-RADS score. If multiple lesions were present with the same PI-RADS score, the lesion with the greatest diameter was considered the index lesion. There was no minimum PI-RADS or size required to be identified as an index lesion. Non-index lesions included all other visible lesions. New lesions on follow-up scans were defined as any lesion that was not previously identified on the previous scan, regardless of size or PI-RADS score.

\section{Statistical analysis}

Our primary end point was to assess for any progression or regression of lesions (both index and non-index), on subsequent scan(s). Lesion progression was defined as either an increase in any diameter by $\geq 2 \mathrm{~mm}$ and/or an increase in the PI-RADS score of an existing lesion. If one parameter increased but the other decreased, the lesion was not considered to have progressed. In contrast, we identified regression as lesions that were no longer visualized on subsequent scans.

In the subgroup of patients on AS, we analyzed the correlation of MRI progression with biopsy reclassification immediately after MRI. In this context MRI progression was defined as lesion progression as above, or the development of a new suspicious lesion (PI-RADS $\geq 4$ ). Biopsy reclassification was defined as diagnosis of a higher Gleason grade or a higher percentage of cores involved with cancer compared to the diagnostic biopsy prior to AS commencement. 
Statistical analysis was performed on IBM SPSS statistics version 22. Independent samples $\mathrm{T}$ test was used for comparison of means, Fisher's exact test for comparison of proportions, and Mann-Whitney $\mathrm{U}$ test for non-parametric independent samples. All tests were two sided with $\mathrm{P}$ values less than 0.5 considered significant.

\section{Results}

Cohort

A total of 83 men who had received multiple scans were selected for analysis. Table 1 summarizes clinical parameters of the men in the AS cohort relative to the diagnosis cohort. The PSA and PSA density were both higher in the diagnosis cohort. Most men had 1 or 2 lesions and it was uncommon to find more than 2 lesions in patients in either group. The average time interval between first and last MRI was 1.9 years.

On the initial scan we identified 66 index lesions and 49 non-index lesions (Table 2). The index lesion was more often located in the peripheral zone than the transitional zone (37.9\% vs. $20.8 \%, \mathrm{p}=0.011$ ). There was no difference in the distribution through apex, mid or base of the prostate.

\section{Lesion progression}

The natural history of index and non-index lesions is summarized in Table 3. The initial size difference between index and non-index lesions was only $0.98 \mathrm{~mm}$. The increase in lesion size, however, was greater among index lesions than non-index lesions, and more index lesions (36.2\%) grew compared to non-index lesions (7.3\%). The mean change in size for all index lesions was $+0.7 \mathrm{~mm}$ over 2 years, whereas the mean for non-index lesions was $-1.34 \mathrm{~mm}$ over the same time span. Approximately one third (39.7\%) of index lesions but two thirds (68.3\%) of non-index lesions did not change in size during the study period.

The PI-RADS score remained the same between initial and follow-up scans in the majority of lesions ( $74.1 \%$ index vs. $87.8 \%$ non-index, $\mathrm{p}=0.158$ ). A similar proportion (12\%) of index and non-index lesions increased to a higher score. Eight out of the 115 total lesions progressed from a PI-RADS $\leq 3$ to a PI-RADS $\geq 4$, four of which were index, and four non-index lesions. Two out of the four index lesions and one out of the four nonindex lesions also increased in size.

Combining size or PIRADS score, 34.8\% index lesions progressed, compared to $25 \%$ non-index lesions. On the other hand $25 \%$ of non-index lesions regressed, compared to $12.1 \%$ index lesions.

Table 4 summarizes findings related to new lesions that were not visualized on initial scans but were identified on follow-up imaging. A total of 79 new lesions were identified in 51/83 men (61.4\%). Of these new index lesions, 13/79 (16.5\%) were PIRADS $\geq 4$. In 29 out of these 51 cases (56.9\%), a new lesion became the new index 
lesion. Eight men developed new PIRADs $\geq 4$ index lesions, with three developing concurrent new $\geq 10 \mathrm{~mm}$ index lesions. Three out of the eight men were diagnosed with prostate cancer after follow-up biopsy. The other five did not receive biopsy. New lesions were more often identified in the apex compared to initial lesions (29.1\% vs. $16 \%$, $\mathrm{p}=0.01)$.

\section{Active surveillance (AS) cohort}

54 men on active surveillance were identified to have received multiple mpMRI. 45/54 (83.3\%) were first diagnosed with prostate cancer after positive cores found on transrectal ultrasound (TRUS) biopsy. 7/54 (13\%) received MRI after prior negative TRUS pathology, despite persistently high PSA and were diagnosed with prostate cancer after receiving fusion biopsy, while 2/54 (3.7\%) were diagnosed based on transurethral resection of the prostate (TURP) pathology.

18 of the 54 (33.3\%) men showed MRI progression. Of these, 13/18 (72.2\%) were not re-biopsied at the discretion of the urologist or due to patient refusal. Three showed biopsy re-classification, and 2 had no biopsy re-classification (Figure 1). Of the 13 patients who were not re-biopsied, 7 (53.8\%) progressed on MRI by size criteria and 1 (7.7\%) by an increase in PI-RADS score, while 5 (38.5\%) demonstrated new suspicious lesions not previously present on prior scans. Four of the patients who were not biopsied terminated AS based on imaging criteria alone, but the other 9 continued on AS.

Of the 36 men who showed a stable subsequent MRI, 21 were not re-biopsied (58.3\%). 7/36 (19.4\%) showed biopsy re-classification (6 Gleason grade increase and 1 increased core involvement), of whom 4/7 (57.1\%) stopped AS. 1/28 (6.7\%) without biopsy re-classification stopped AS (PI-RADS progression detected under prior version which was used at the time of this patient's MRI). Overall, in the MRI progression cohort, we saw $33.3 \%$ of men terminate AS, whereas only $13.9 \%$ stopped AS in the MRI stable group $(\mathrm{p}=0.09)$.

\section{Discussion}

A key unmet clinical need in the detection of prostate cancer, and on the comprehensive characterization of supposed low risk lesions on active surveillance, has been our inability to visualize the cancer on standard imaging modalities. Instead we have been dependent on systematic biopsies carried out in relatively blind fashion, PSA kinetics and digital rectal exam $(1,2,5,14,15)$. Multiparametric MRI, however, has dramatically shifted this paradigm, and is allowing us now to assess tumor burden more accurately, and target lesions for biopsy that were previously not appreciated by available clinical tools $(1,15$, 16)

With the increased use of MRI in prostate cancer detection and in men on active surveillance, serial imaging of the prostate is becoming a more common clinical practice. 
The natural history of prostatic lesions on serial multiparametric MRI is not well described in the literature. While many studies have shown the benefits of MRI imaging, very little information is available regarding changes in lesion characteristics on serial imaging. Stevens et al. (17) reported in abstract form on MRI lesion progression after only a 3-month interval in 98 men on AS. Of these, 14 men terminated AS due to a more concerning lesion on the second MRI. In this very limited follow-up period, they found that the majority of lesions (52\%) remained stable and only a small minority showed signs of progression. Although they did not specify index vs. non-index lesions, they found lesion progression to be more likely among men with an obvious lesion at baseline.

More recently, Felker et al. (18) studied the natural history of prostate cancer on AS through serial MRI. They looked at 48 men with an index lesion on baseline MRI who received a $2^{\text {nd }}$ MRI along with a targeted prostate biopsy. They found that 10 patients (20.8\%) had MRI progression of an index lesion after a mean of 2.4 years, compared to our findings of $34.8 \%$ after 1.9 years. While we assessed index lesion progression based on PI-RADS score and lesion diameter, this study defined index lesions based on volume.

Our study has demonstrated some important findings with regard to the natural history of MRI lesions. The mean increase in size of index lesions in the 1.9 year study period was $0.7 \mathrm{~mm}$. 34.8\% of index lesions, but only $7.6 \%$ of non-index lesions progressed, as defined by size and/or PI-RADS score. New lesions were identified in $61 \%$ of patients, and these new lesions became the index lesion in $34.9 \%$ of cases. Overall these findings confirm what one would expect with serial MRI, which is that there is a very slow natural history of prostate lesions identified on MRI in patients selected as they were here. The patients represented here are those without a diagnosis of prostate cancer who had a prior negative biopsy and had an MRI that was determined suitable for further observation with a follow-up MRI, and those on active surveillance. In both instances, we have selected relatively low risk patients, and we cannot draw any conclusions on the timeline of lesion evolution in intermediate and high risk cancers.

Our data provide some guidance on choosing the interval between MRI scans in men in the described clinical settings. There is little information in the literature pertaining to the appropriate amount of time between scans. Our findings suggest that repetitive scans within short time intervals is likely not helpful. This is in line with findings from RaisBahrami et al. (19) who suggested that patients with small index lesions harboring insignificant disease as determined by fusion biopsy, demonstrate minimal change for at least two years, and therefore do not require continuous testing and monitoring within that period of time. Their study further suggested conducting active surveillance screening every two-years, which is consistent with our results. Our findings do not apply to higher risk situations, but patients with higher risk lesions are unlikely to be under surveillance with follow-up imaging. 
Only one third of our AS cohort showed MRI progression. Key clinical decisions were made based on MRI findings including the timing of follow-up biopsy and the need to move to definitive intervention. However, since re-classification was seen in both MRI stable and MRI progression cohorts, we can infer that biopsies are still a key determinant in making treatment decisions.

Limitations of this study include especially the retrospective nature of the study and the relatively small sample size. The introduction of PI-RADS 2.0 in the midst of our study is a confounding factor, since some clinical decisions were made based on the prior PI-RADS version. We have compared only two scans in series for the vast majority of patients, but longer follow-up with more scans would provide more information on the natural history of these MRI lesions. Further, the use of a single radiologist limited interrater agreement of the scans. Selection bias in determining which patients underwent surveillance of their MRI lesions versus intervention (e.g. treatment of prostate cancer if on AS or targeted biopsy if in diagnostic cohort) limits the generalizability of the findings. The subjective use of MRI for management decisions in patients on AS will be different between individual physicians and different centers. Further subjective measures such as urologist and/or patient preference, prevented standardized confirmatory biopsy in both MRI stable and progression cohorts. There are no standard criteria to define progression on MRI, so our definitions are to some degree arbitrary. This was done due to the importance of having some margin definition to correct for irrelevant differences due to human error in measuring. Furthermore, we acknowledge that our definition of index lesion encompassed lesions of different characteristics, and that some index lesions were more clinically significant than others at the time of discovery. We are unable to provide histologic findings for each lesion based on fusion biopsy, which would offer the most comprehensive characterization. We acknowledge that most centers have moved to 3.0T MRI, and that our practice of using 1.5T imaging without endorectal coil appear unconventional, but we have published previous results in this domain that do not differ significantly from other series $(1,2)$. Further, we analyzed patients on active surveillance along with non-tissue proven patients with rising PSA. While we recognize the arbitrary nature of this selection, our goal remained to describe changes in lesions over time, regardless of clinical context.

\section{Conclusion}

Our findings suggest that index lesions on MRI are much more likely to progress over time than non-index lesions. However, changes in size and PI-RADS scoring were generally small over the 1.9 year study period. This suggests that frequent tests are not necessary to assess cancer growth in these patient populations, with low-risk disease. Though new lesions were common on follow-up scans, the majority were not of clinical significance in the short term and did not alter patient management. A small minority of 
men terminated active surveillance due to MRI progression. However, biopsy reclassification continued to play a crucial role in determining the clinical course of patients both with stable MRI findings and with lesion progression on MRI. A larger prospective study is needed to determine the true role of serial imaging plays on termination of AS. 


\section{References}

1. Abdi H, Pourmalek F, Zargar H, et al. Multiparametric magnetic resonance imaging enhances detection of significant tumor in patients on active surveillance for prostate cancer. Urology. 2015;85:423-428.

2. Abdi H, Zargar H, Goldenberg SL, et al. Multiparametric magnetic resonance imaging-targeted biopsy for the detection of prostate cancer in patients with prior negative biopsy results. Urol Oncol. 2015;33:165.e161-167.

3. Hodge KK, McNeal JE, Stamey TA. Ultrasound guided transrectal core biopsies of the palpably abnormal prostate. J Urol. 1989;142:66-70.

4. Barentsz JO, Richenberg J, Clements R, et al. ESUR prostate MR guidelines 2012. Eur Radiol. 2012;22:746-757.

5. Cooperberg MR, Carroll PR, Klotz L. Active surveillance for prostate cancer: progress and promise. J Clin Oncol. 2011;29:3669-3676.

6. Ahmed HU, Bosaily AE, Brown LC, et al. Diagnostic accuracy of multiparametric MRI and TRUS biopsy in prostate cancer (PROMIS): a paired validating confirmatory study. Lancet. 2017;389:815-822.

7. Halliwell OT, Yadegafar G, Lane C, et al. Transrectal ultrasound-guided biopsy of the prostate: aspirin increases the incidence of minor bleeding complications. Clin Radiol. 2008;63:557-561.

8. Loeb S, Vellekoop A, Ahmed HU, et al. Systematic review of complications of prostate biopsy. Eur Urol. 2013;64:876-892.

9. Wagenlehner FM, van Oostrum E, Tenke P, et al. Infective complications after prostate biopsy: outcome of the Global Prevalence Study of Infections in Urology (GPIU) 2010 and 2011, a prospective multinational multicentre prostate biopsy study. Eur Urol. 2013;63:521-527.

10. Zaytoun OM, Anil T, Moussa AS, et al. Morbidity of prostate biopsy after simplified versus complex preparation protocols: assessment of risk factors. Urology. 2011;77:910-914.

11. Weinreb JC, Barentsz JO, Choyke PL, et al. PI-RADS Prostate Imaging Reporting and Data System: 2015, Version 2. Eur Urol. 2015.

12. Carter HB, Partin AW, Walsh PC, et al. Gleason score 6 adenocarcinoma: should it be labeled as cancer? J Clin Oncol. 2012;30:4294-4296.

13. Ahmed HU, Arya M, Freeman A, et al. Do low-grade and low-volume prostate cancers bear the hallmarks of malignancy? Lancet Oncol.2012;13:e509-517.

14. Fascelli M, George AK, Frye T, et al. The role of MRI in active surveillance for prostate cancer. Curr Urol Rep. 2015;16:42. 
15. Siddiqui MM, Rais-Bahrami S, Truong H, et al. Magnetic resonance imaging/ultrasound-fusion biopsy significantly upgrades prostate cancer versus systematic 12-core transrectal ultrasound biopsy. Eur Urol. 2013;64:713-719.

16. Walton Diaz A, Shakir NA, George AK, et al. Use of serial multiparametric magnetic resonance imaging in the management of patients with prostate cancer on active surveillance Urol Oncol. 2015;33:202.e201-207

17. Stevens DJ, Moore C, Ahmed H, et al. 1096 the natural history of untreated prostate MRI lesions in an active surveillance prostate cancer population-260 isep:patient-years. Eur Urol Suppl 2012; 11:; e1096-e1096a.

18. Felker ER, Wu J, Natarajan S, et al. Serial Magnetic Resonance Imaging in Active Surveillance of Prostate Cancer: Incremental Value J Urol 2016;195:14211427

19. Rais-Bahrami S, Türkbey B, Rastinehad AR, et al. Natural history of small index lesions suspicious for prostate cancer on multiparametric MRI: recommendations for interval imaging follow-up. Diagn Interv Radiol. 2014;20:293-298. 


\section{Figures and Tables}

Fig. 1. Active surveillance - schematic depicting disease re-classification and termination of active surveillance after serial magnetic resonance imaging (MRI) monitoring. The mean interval between MRI scans was 1.9 years.

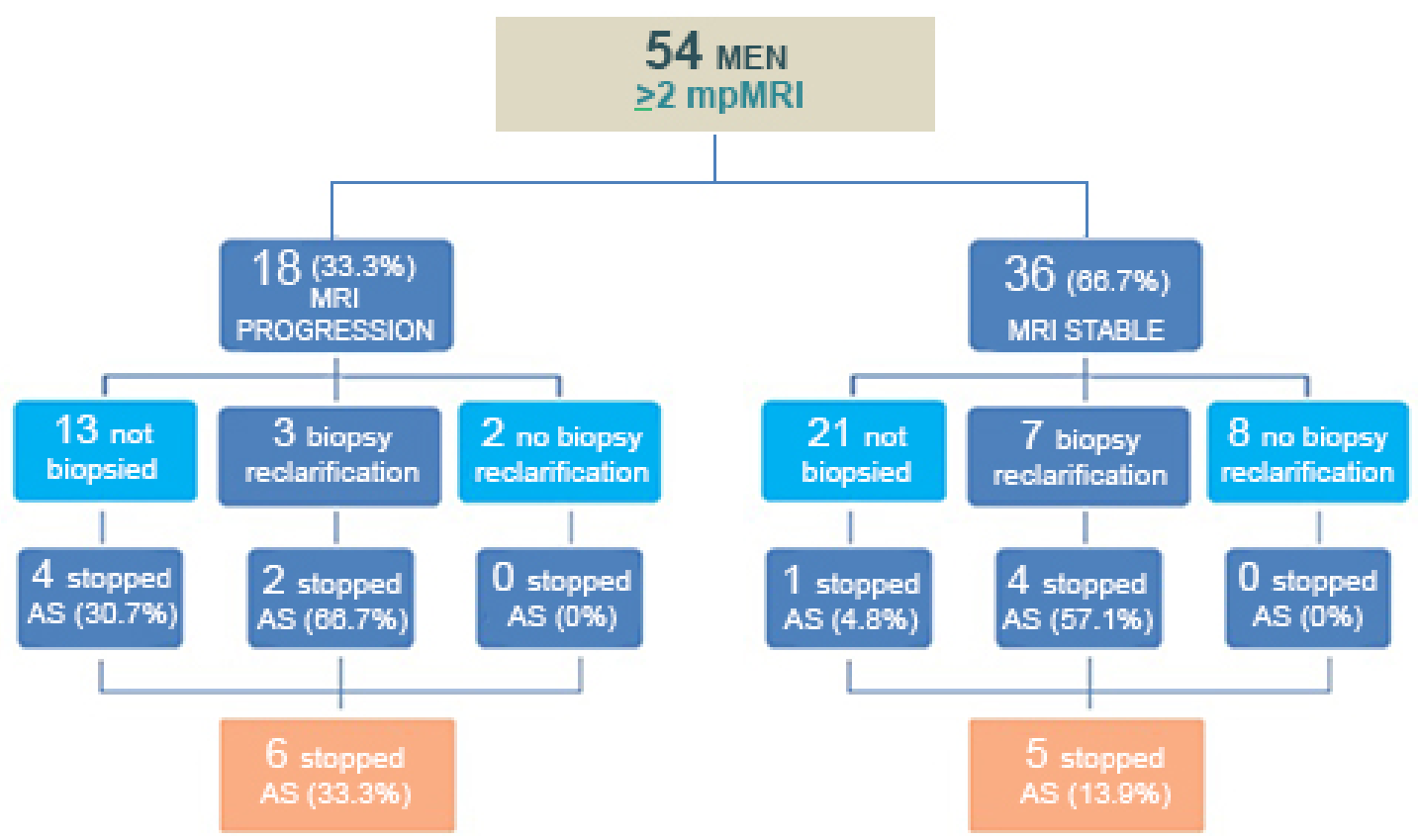


Table 1. Clinical characteristics of patient cohorts undergoing serial magnetic resonance imaging (MRI)

\begin{tabular}{|c|c|c|c|c|}
\hline & Cohort $(n=83)$ & Active Surv. $(n=54)$ & Diagnosis $(n=29)$ & P-value \\
\hline $\begin{array}{l}\text { Age } \\
- \text { Median (IQR) }\end{array}$ & $64.3(59.0-69.8)$ & $65.7(60.2-69.9)$ & $63.0(58.4-67.4)$ & 0.401 \\
\hline $\begin{array}{l}\text { Prostate Volume } \\
\text { - Median (IQR) }\end{array}$ & $48.0(33.0-75.0)$ & $48.5(33.8-74.3)$ & $47.0(30.0-89.0)$ & 0.397 \\
\hline $\begin{array}{l}\text { PSA (ng/mL) } \\
-\quad \text { Median (IQR) }\end{array}$ & $7.0(5.0-10.0)$ & $5.8(4.2-7.3)$ & $9.6(7.9-12.5)$ & $<0.001$ \\
\hline $\begin{array}{l}\text { PSA density } \\
\text { - Median (IQR) }\end{array}$ & $0.12(0.07-0.22)$ & $0.10(0.06-0.15)$ & $0.22(0.11-0.30)$ & 0.017 \\
\hline $\begin{array}{l}\# \text { of lesions on } 1^{\text {st }} \text { scan } \\
-\quad 0 \\
-1 \\
-2 \\
-3 \\
-4 \\
-5\end{array}$ & $\begin{array}{c}17(20.5) \\
29(34.9) \\
30(36.1) \\
5(6.0) \\
1(1.2) \\
1(1.2)\end{array}$ & $\begin{array}{c}12(22.2) \\
20(37.0) \\
17(31.5) \\
4(7.4) \\
1(1.9) \\
0\end{array}$ & $\begin{array}{c}5(17.2) \\
9(31.0) \\
13(44.8) \\
1(3.4) \\
0 \\
1(3.4)\end{array}$ & 0.515 \\
\hline $\begin{array}{l}\text { MRI observation time (yrs) } \\
-\quad \text { Median (IQR) }\end{array}$ & $1.87(1.14-2.74)$ & $1.89(1.18-2.74)$ & $1.84(1.03-2.63)$ & 0.936 \\
\hline
\end{tabular}

IQR: interquartile range; PSA: prostate-specific antigen. 
Table 2. Characteristics of index lesions vs. non-index lesions in 83 men followed with serial magnetic resonance imaging (MRI) of the prostate

\begin{tabular}{|c|c|c|c|}
\hline & Index lesions & Non-Index lesions & P-value \\
\hline Total (n) & 66 & 49 & \\
\hline \multicolumn{4}{|l|}{ Size $(\mathrm{mm})$} \\
\hline - Median & 11 & 10 & 0.130 \\
\hline Range & $5-30$ & $4-29$ & \\
\hline \multicolumn{4}{|l|}{ PIRADS (V2.0) } \\
\hline-1 & $4(6.1)$ & $6(12.2)$ & 0.004 \\
\hline 2 & $33(50.0)$ & $32(65.3)$ & \\
\hline 3 & $18(27.3)$ & $11(22.4)$ & \\
\hline 4 & $8(12.1)$ & 0 & \\
\hline 5 & $3(4.5)$ & 0 & \\
\hline \multicolumn{4}{|l|}{ Location (\%) } \\
\hline - Apex & $8(12.1)$ & $11(22.4)$ & 0.077 \\
\hline Mid & $33(50)$ & $21(42.8)$ & \\
\hline - Base & $25(37.9)$ & $17(34.6)$ & \\
\hline \multicolumn{4}{|l|}{ Zone } \\
\hline - Peripheral & $25(37.9)$ & $10(20.8)$ & 0.011 \\
\hline Transition & $41(62.1)$ & $38(79.1)$ & \\
\hline
\end{tabular}

PIRADS: Prostate Imaging Reporting and Data System. 
Table 3. Natural history of index and non-index lesions

\begin{tabular}{|c|c|c|c|}
\hline & Index Lesions ( $n=66$ ) & Non-Index Lesions $(n=49)$ & P value \\
\hline Time Followed (yrs) & & & \\
\hline - Median & 1.75 & 1.42 & 0.512 \\
\hline -Range & $0.4-4.99$ & $0.4-3.7$ & \\
\hline - Mean \pm SD & $1.87 \pm 0.97$ & $1.66 \pm 0.77$ & 0.197 \\
\hline $\begin{array}{l}\text { Lesion Size on Initial } \\
\text { MP-MRI (mm) }\end{array}$ & $12.74 \pm 5.40$ & $11.76 \pm 6.21$ & 0.149 \\
\hline $\begin{array}{l}\text { Lesion Size on Most } \\
\text { Recent MP-MRI (mm) }\end{array}$ & $13.45 \pm 5.58$ & $10.42 \pm 5.37$ & 0.010 \\
\hline $\begin{array}{l}\text { Size Change } \\
\text { - Decrease } \\
\text { - Stable }( \pm 2 \mathrm{~mm}) \\
\text { - Increase }\end{array}$ & $\begin{array}{l}14(24.1) \\
23(39.7) \\
21(36.2)\end{array}$ & $\begin{array}{l}10(24.4) \\
28(68.3) \\
3(7.3)\end{array}$ & 0.002 \\
\hline $\begin{array}{l}\text { PI-RADS Change } \\
\text { - Decrease } \\
\text { - Stable } \\
\text { - Increase }\end{array}$ & $\begin{array}{l}3(5.2) \\
43(74.1) \\
12(20)\end{array}$ & $\begin{array}{l}0 \\
36(87.8) \\
5(12.2)\end{array}$ & 0.158 \\
\hline $\begin{array}{l}\text { Progressed (Size or } \\
\text { PI-RADS increased }\end{array}$ & $23(34.8)$ & $4(7.6)$ & $<0.001$ \\
\hline $\begin{array}{l}\text { Regressed (No longer } \\
\text { visible) }\end{array}$ & $8(12.1)$ & $12(25)$ & 0.115 \\
\hline
\end{tabular}

mpMRI: multiparametric magnetic resonance imaging; PIRADS: Prostate Imaging Reporting and Data System; SD: standard deviation. 
Table 4. Comparison of features of existing lesions vs. new lesions identified on serial imaging

\begin{tabular}{|c|c|c|c|}
\hline & $\begin{array}{l}\text { Lesion Visible on Previous } \\
\text { MRI }\end{array}$ & $\begin{array}{l}\text { New Lesion Not Visible on } \\
\text { Previous MRI }\end{array}$ & P-Value \\
\hline a. Number & 115 & 79 & - \\
\hline Size $(\mathrm{mm})$ & $12.28 \pm 5.68$ & $10.81 \pm 4.56$ & 0.048 \\
\hline \multicolumn{4}{|l|}{ Zone } \\
\hline - Peripheral & $36(30.3)$ & $26(32.9)$ & 0.294 \\
\hline Transition & $79(66.3)$ & $52(65.8)$ & \\
\hline Anterior & 0 & $1(1.3)$ & \\
\hline \multicolumn{4}{|l|}{ Location } \\
\hline Apex & $19(16.0)$ & $23(29.1)$ & 0.010 \\
\hline Mid & $54(45.4)$ & $41(51.9)$ & \\
\hline Base & $42(35.3)$ & $15(19)$ & \\
\hline \multicolumn{4}{|l|}{ PIRADS (V2.0) } \\
\hline 1 & $10(8.7)$ & $5(6.3)$ & 0.122 \\
\hline 2 & $65(56.5)$ & $46(58.2)$ & \\
\hline 3 & $29(25.2)$ & $15(19.0)$ & \\
\hline 4 & $8(7.0)$ & $13(16.5)$ & \\
\hline 5 & $3(2.6)$ & 0 & \\
\hline
\end{tabular}

MRI: magnetic resonance imaging; PIRADS: Prostate Imaging Reporting and Data System. 